\title{
Radiation-induced Tumorigenesis
}

\author{
In-Gyu Kim and Yun-Sil Lee ${ }^{\dagger *}$ \\ Department of Radiation Biology, Environmental Radiation Research Group, Korea Atomic Energy Research Institute, \\ Taejon 305-600, Korea \\ Laboratory of Radiation Effect, Korea Institute of Radiological and Medical Sciences, Seoul 139-706, Korea
}

Received 31 October 2002

\begin{abstract}
During the past 2 decades, radiation tumorigenesis researchers have focused on cellular and molecular mechanisms. We reviewed some of these research fields, since they may specifically relate to the induction of cancer by ionizing radiation. First, radiation-mediated mutation was discussed. Then the initiating event in radiation carcinogenesis, as well as other genetic events that may be involved, is discussed in terms of the possible role of the activation of genes and the loss of cell-cycle checkpoints.
\end{abstract}

Keywords: Cell cycle, Gene Expression, Initiation, Mutation, Radiation, Tumorigenesis

\section{Introduction}

The carcinogenic effect of ionizing radiation was recognized just after Roentgens discovery of X-ray in 1895. The first radiationinduced cancer was reported to arise in an ulcerated area of the skin. Within a few years, a large number of these skin cancers were observed. The first report of leukemia, occurring in five radiation workers, appeared in 1911 (Upton, 1986).

Animal models to study radiation carcinogenesis were primarily developed after World War II. Large-scale tumor induction studies were carried out in mice and rats over the next 3 decades (Fry and Storer, 1987). These studies defined many of the general characteristics of radiation carcinogenesis, and were supported by emerging findings from various human epidemiologic studies that received radiation exposure from occupational, medical, and accidental sources. Among these, the long-term follow-up of the atom bomb survivors from Hiroshima and Nagasaki is foremost (Pierce et al., 1989). These studies show radiation as a universal carcinogen, since it induces cancer in most tissues of

*To whom correspondence should be addressed.

Tel: 82-2-970-1325; Fax: 82-2-977-0381

E-mail: yslee@kcch.re.kr most species at all ages, including the fetus. The cancers that are induced by radiation are of the same histological types as the ones that occur spontaneously, but the distribution of the types may differ. Finally, it became evident that radiation carcinogenesis can be modulated by a variety of noncarcinogenic secondary factors.

The universal nature of radiation as a carcinogen relates to a specific characteristic of ionizing radiation that differentiates it from chemical toxic agents or other physical carcinogens, which are usually tissue specific in their action. This shows its ability to penetrate cells and to deposit energy within them in a random fashion, unaffected by the usual cellular barriers that are presented to chemical agents. All of the cells in the body are thus susceptible to damage by ionizing radiation; the amount of damage will be related to the physical parameters that determine the radiation dose that is received by the particular cells or tissue. Cellular systems were developed in the 1970s to study the malignant transformation of individual cells in vitro (Chadwick et al., 1989; Little, 1989). The findings in these studies confirmed several of the conclusions that were derived from animal studies.

By 1980, therefore, the general characteristics of radiation that induced cancer in vivo and of cellular transformation in vitro were well established. In fact, only a limited work has proceeded in these areas since that time. Rather, research during the past 2 decades has focused on the cellular and molecular mechanisms for the effects of radiation in mammalian cells. In fact, ionizing radiation has become a widely-used tool for studying the cellular and molecular response to DNA damage, as these serve to enhance our understanding of the carcinogenic process itself.

To complicate the picture, accumulations of mutant genes in neoplasia tend to be accompanied by other genetic and epigenetic changes. These changes include the loss of heterozygosity, inactivation of important genes by methylation or loss of imprinting, and gene amplification. All of these can alter gene expression profiles. Therefore, the genome-wide monitoring of the gene expression is of great importance if we are to discover the numerous and diverse events that are 


\section{Mutation spectrum of $h p r t$ by PCP}

\section{Base 190 GC-CG (transversion) $20.7 \%$ \\ Base $444 \quad---$. CG (insertion) 17.2\%}

\section{Mutation spectrum of hprt by radiation}

\section{E1-E9 Large deletion $75 \%$}
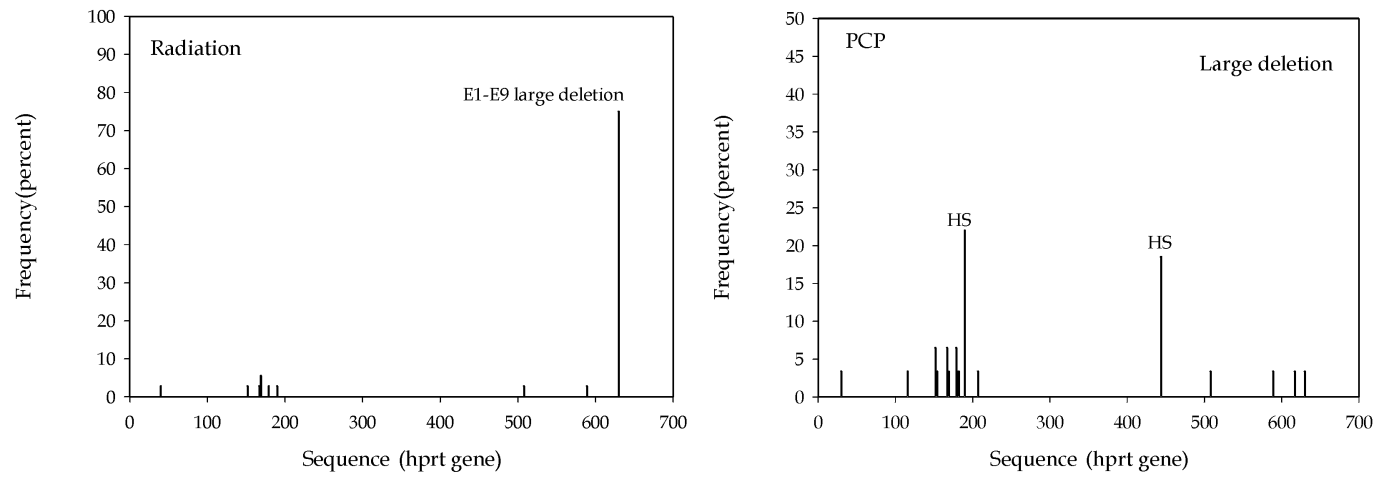

Fig. 1. Mutation spectra of the hprt gene when treated with radiation or pentachlorophenol (PCB) in human peripheral lymphocytes. Human T-cell hprt clonal assay and hypoxanthine (guanine) phosphoribosyl transferase methods were used.

associated with carcinogenesis.

In this review, we discussed several research areas that may specifically relate to the induction of cancer by ionizing radiation.

\section{Radiation-induced Mutation}

Although the mutagenic potential of radiation was first described by Muller in 1927, only within the past 2 decades has this information gained interest concerning the molecular structure of mutations in mammalian cells. Our studies with the hemizygous X-linked hprt gene showed that radiation could induce both point mutations and deletions (Fig. 1); the latter sometimes includes the entire gene (Tracker, 1986). However, the size of the deletions at a hemizygous locus that can give rise to a viable cell is limited, owing to the nearby presence of essential genes and mutations that cannot be induced by homologous-recombinational processes. When a system was developed to study the mutational events at an autosomal locus where both copies of the gene were present, then large-scale events that involved the loss of heterozygosity $(\mathrm{LOH})$ were found to be the most frequent ones (Yandell et $a l ., 1986)$. This LOH was frequently extended to include other genes on the chromosome, both proximal and distal to the target gene (Li et al., 1992). Although LOH was often the result of a simple deletion, it was shown, at least in some cases, to be the result of recombinational processes (Yandell et

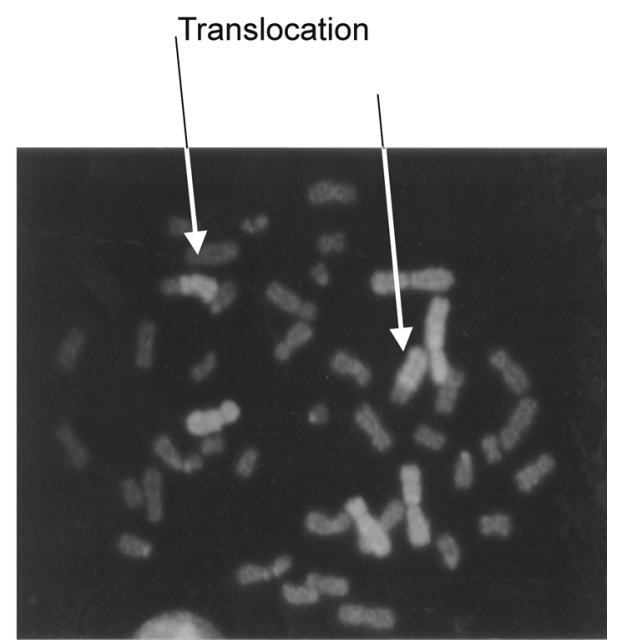

Fig. 2. Chromosome aberration by detected fluorescence in situ hybridization (FISH).

\section{al., 1986; Li et al., 1992).}

Therefore, the predominant molecular-structural changes that are associated with radiation-induced mutations are largescale events, including $\mathrm{LOH}$ of the gene that may extend to other loci on the chromosome. These large-scale events include deletions, chromosomal rearrangements, or recombinational processes; these mutational changes are of the type that one would expect to arise as a consequence of double-strand breaks (DSBs). 


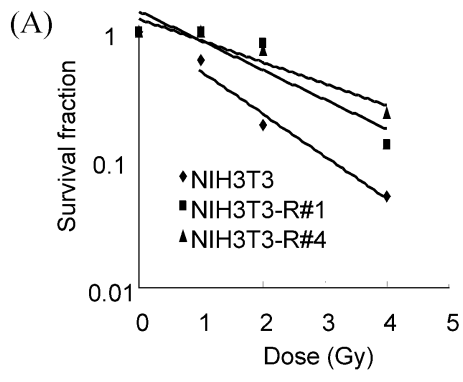

(C)

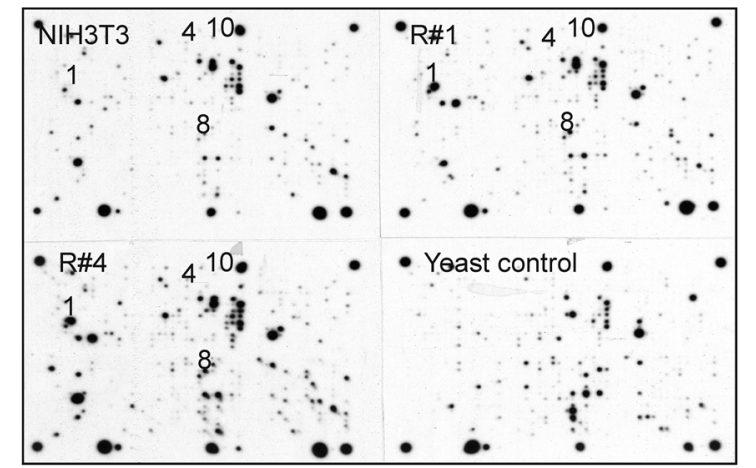

(B) NIH3T3 NIH3T3-\#1 NIH3T3-\#4

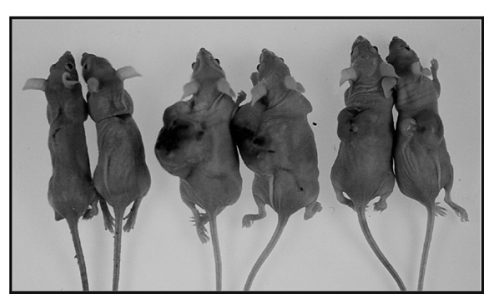

(D)

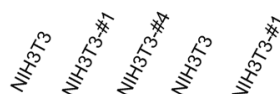

Cdk6

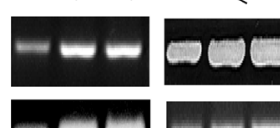

$\mathrm{Mdm} 2$

Cdc25B

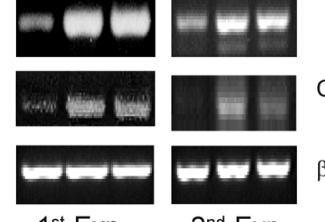

$1^{\text {st }} \operatorname{Exp} \quad 2^{\text {nd }} \operatorname{Exp}$

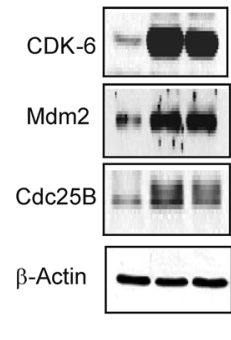

1: cdk6 4: mdm2 8: cdc25B 10: entactin (nidogen 1)

Fig. 3. (A) Selection of radiation-resistant clones in NIH3T3 cells by clonogenic survival (B). Tumorigenesis of radiation-resistance clones when grafted to nude mice (C). Altered gene expression of microarray in radiation-resistant clones (D). Confirmation of results from macroarray analysis using RT-PCR and Western blotting.

\section{Initiating Event in Radiation Carcinogenesis}

The search for genetic changes that are specially associated with radiation exposure has proven very disappointing. There is no evidence of site specificity for mutations that are induced by radiation. The spectrum of molecular-structural changes that are associated with direct radiation-induced mutations markedly differs from that for spontaneous mutations. In the latter case, point mutations predominate. Figure 1 graphically shows the X-linked hprt gene. However, there appears to be no site specificity for DNA break points that lead to deletions. Similarly, sequence analyses of radiation-induced point mutations have generally shown no site specificity (Grosovsky et al., 1988). Furthermore, no genetic alterations that are unique to radiation have as yet been found in radiationinduced tumors.

It is well known that radiation can induce a wide variety of stable chromosomal aberrations, including deletions and reciprocal translocations (Fig. 2). It is tempting to speculate that they may play a more fundamental role in the process of radiation carcinogenesis. Cancer cells are typically aneuploid and contain multiple chromosomal changes. In some cases, specific chromosomal abnormalities have been associated with specific tumor types, such as some leukemias. While radiation-induced cancers show multiple unbalanced chromosomal rearrangements, few show specific translocations or deletions as would be associated with the activation of known oncogenes or tumor suppressor genes
(Dutrillaux, 1997).

Unbalanced translocations can lead to deletions. Certain cancers that are known to be induced by radiation are those in which deletions occur. It has been proposed that the most likely mutational event in the initiation of radiation carcinogenesis involves $\mathrm{LOH}$ of the tumor suppressor gene (Dutrillaux, 1997). One specific example of this phenomenon is the RB tumor suppressor gene that is located in chromosome 13q14. The hypersensitivity of retinoblastoma patients to the induction of secondary cancers (primarily osteosarcomas in the irradiated field following radiotherapy) is presumably the result of radiation-induced $\mathrm{LOH}$ of the RB genes. If this is true, then there must be multiple, although as yet unrecognized, genes that are involved. Although knockout mice that are heterozygous for the p53 tumor suppressor gene are more susceptible to radiation-induced tumors, evidence suggests that $\mathrm{LOH}$ at this locus may not be the initiating event (Bouffler et al., 1995). In this context, it is notable that the expression of p53 mutations also appears to occur late in the process of radiation-induced malignant transformation, during the growth of the visible-transformed foci, an observation that is consistent with the findings in some human tumors (Offner et al., 1999).

While the involvement of various oncogenes in experimental and human carcinogenesis is well established, no data have emerged from animal models to suggest a general role for oncogene activation in radiation-induced cancer. The activation of RAS family members has been 
(A)

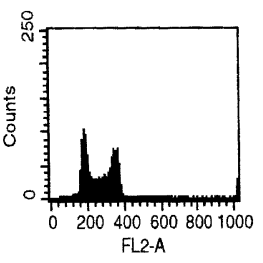

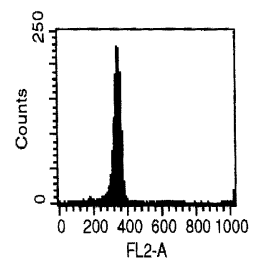

G2 arrest

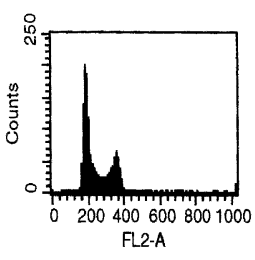

G1 arrest
(B)

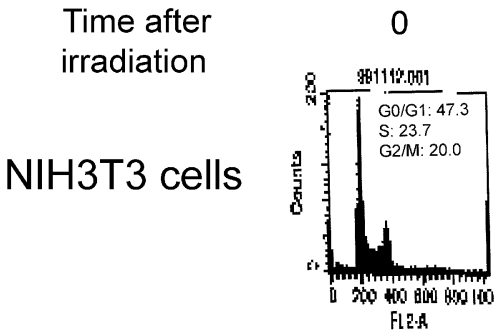

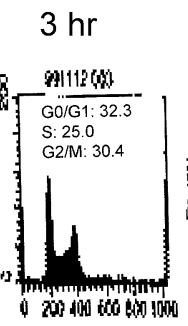

$6 \mathrm{hr}$

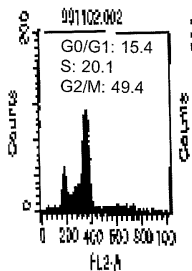

Hath
$12 \mathrm{hr}$

$24 \mathrm{hr}$

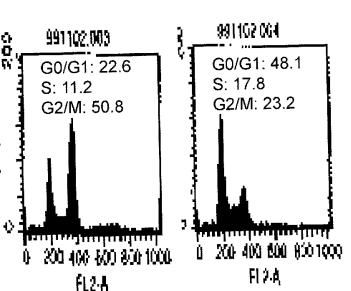

Fig. 4. (A) Radiation-induced G1 (24 hr) or G2 (12 hr) cell cycle arrest in L929 cells. (B) Cell cycle distribution after irradiation in NIH3T3 cells.

reported in a small fraction of certain mouse lymphomas. Amplification and rearrangement of C-MYC was found in a small percentage of radiation-induced murine osteosarcomas. To my knowledge, however, these scattered early results are inconsistent, though it is clear that the pattern of oncogene activation differs significantly for carcinogenesis that is induced by chemical carcinogens when compared to radiation. Recently, we investigated to see if radiation that induced tumorigenic cells showed different gene expression files in the cDNA microarray system, and whether MDM-2 and CDC25B was involved in these phenomena (Fig. 3, unpublished data).

The existence of cell-cycle checkpoints was first demonstrated by a number of radiobiologists in the 1960s. Most prominent among these were a radiation-induced reversible $\mathrm{G}_{2} / \mathrm{M}$ delay that was observed in essentially all types of mammalian cells, and $\mathrm{G}_{1} / \mathrm{S}$ delay that was primarily observed in normal diploid cells (Fig. 4). Since most of these studies were carried out with either transformed rodent or human tumor cell lines, this early research focused on the $G_{2}$ checkpoint as a possible initiating event in radiation carcinogenesis.

In the mid-1980s it was first described that following irradiation of normal human diploid fibroblast cultures, many of the cells were irreversibly arrested at the $G_{1} / S$ border and appeared to enter a senescent-like state (Little and Nagasawa, 1985). When the cells were held in confluence for several hours after irradiation in order to allow the repair of potentially lethal radiation damage, then the fraction of cells that were arrested in $G_{1}$ was reduced in a dose-dependent manner. These findings led to the hypothesis that such an irreversible $G_{1}$ arrest may serve as a mechanism for the elimination of heavily-damaged, and thus potentially-mutated,

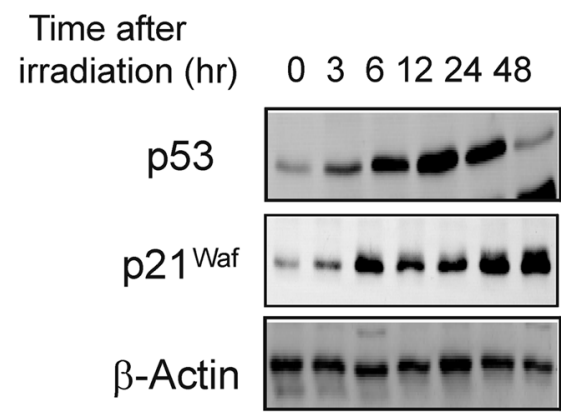

Fig. 5. Radiation-induced p53 and p $21^{\text {Waf }}$ proteins.

cells from the irradiated population. This represents a mechanism that was proposed for cell types, such as those of lymphoid or hematopoietic origin, that readily undergo apoptotic cell death as a consequence of radiation exposure. The occurrence of this irreversible $G_{1}$ arrest, as well as the reversible $G_{1}$ delay that occurs in the remaining cells, was subsequently shown to be dependent upon the wild-type p53 protein expression, owing to the activation of its downstream effector p21 ${ }^{\text {wafl }}$ (Fig. 4).

These results, therefore, suggest that the loss of a normal checkpoint control in itself is not likely to be a principleinitiating event in radiation carcinogenesis. This is consistent with the lack of evidence for the specific involvement of p53 mutations in malignant transformation or radiation-induced tumors. Indeed, when p53 mutations do occur, they appear to be a late event in these processes.

Acknowledgments This work was supported by the Nuclear R\&D Program from the Ministry of Science and Technology of Korea. 


\section{References}

Bouffler, S. D., Kemp, C. J., Balmain, A. and Cox, R. (1995) Spontaneous and ionizing radiation-induced chromosomal abnormalities in p53-deficient mice. Cancer Res. 55, 38833889.

Chadwick, K. H., Seymour, C. and Barnhart, N. (1989) Cell Transformation and Radiation-induced Cancer, Adam Hilger, New York.

Dutrillauz, B. (1997) Ionizing radiation induced malignancies in man. Radioprotection. 32, C431-C440.

Fry, R. J. M. and Storer, J. B. (1987) External radiation carcinogenesis: in Advances in Radiation Biology, Lett, J. T. (ed.), Vol. 13, pp. 31-90, Academic Press, New York.

Grosovsky, A. J., de Noer, J. G., de Jong, P. J., Drobetsky, E. A. and Glickman, B. W. (1988) Base substitutions, frameshifts and small deletions constitute ionizing radiation-induced point mutations in mammalian cells. Proc. Natl. Acad. Sci. USA 85, 185-1988

Li, C. Y., Yandell, D. W. and Little, J. B. (1992) Molecular mechanisms of spontaneous and induced loss of heterozygosity in human cells in vivo. Somat. Cell. Mol. Genet. 7, 77-87.

Little, J. B. (1989) The relevance of cell transformation to carcinogenesis in vivo: in Low Dose Radiation-Biological Basis of Risk Assessment, Baverstock, K. F. and Strather, J. W. (eds.), pp. 396-413, Taylor and Francis, London, UK.

Little, J. B. and Nagasawa, H. (1985) Effect of confluent holding on potentially lethal damage repair, cell cycle progression and chromosomal aberrations in human normal and ataxiatelangienctasia fibroblast. Radiat. Res. 101, 81-93.

Offner, S., Schmaus, W., Witter, K., Baretton, G. B., Schlimok, G., Passlick, B., Reithmuller, G. and Pantel, K. (1999) p53 gene mutations are not required for early dissemination of cancer cells. Proc. Natl. Acad. Sci. USA 96, 6942-6946.

Pierce, D., Shimizu, Y., Preston, D., Vaeth, M. and Maubuchi, K. (1996) Studies of the mortality of atomic bomb survivors. Radiat. Res. 146, 1-27.

Tracker, J. (1986) The nature of mutants induced by ionizing radiation in cultured hamster cells. Mutat. Res. 160, 267-275.

Upton, A. C. (1986) Historical perspective on radiation carcinogenesis: in Radiation Carcinogenesis, Upton, A. C., Alber, R. E., Burns, F. J. and Shore, R. E. (eds.), pp. 1-10, Elsevier, New York.

Yandell, D. W., Dryup, T. P. and Little, J. B. (1986) Somatic mutations at a heterozygous autosomal locus in human cells occur more frequently by allele loss than by intragenic structure alterations. Somat. Cell. Mol. Genet. 12, 255-263. 